\title{
Perfusion Deficits and Mismatch in Patients with Acute Lacunar Infarcts Studied with Whole-Brain CT Perfusion
}

\author{
S. Rudilosso, X. Urra, L. San Román, C. Laredo, A. López-Rueda, S. Amaro, L. Oleaga, and Á. Chamorro
}

\begin{abstract}
BACKGROUND AND PURPOSE: The incidence and significance of perfusion abnormalities on brain imaging in patients with lacunar infarct are controversial. We studied the diagnostic yield of CTP and the type of perfusion abnormalities in patients presenting with a lacunar syndrome and in those with MR imaging-confirmed lacunar infarcts.
\end{abstract}

MATERIALS AND METHODS: A cohort of 33 patients with lacunar syndrome underwent whole-brain CTP on admission. Twenty-eight patients had an acute ischemic lesion at follow-up MR imaging; 16 were classified as lacunar infarcts. Two independent readers evaluated NCCT and CTP to compare their diagnostic yield. In patients with DWI-confirmed lacunar infarcts and visible deficits on CTP, the presence of mismatch tissue was measured by using different perfusion thresholds.

RESULTS: The symptomatic acute lesion was seen on CTP in 50\% of patients presenting with a lacunar syndrome compared with only $17 \%$ on NCCT, and in $62 \%$ on CTP compared with $19 \%$ on NCCT, respectively, in patients with DWI-confirmed lacunar infarcts. CTP was more sensitive in supratentorial than in infratentorial lesions. In the nonblinded analysis, a perfusion deficit was observed in 12/16 patients with DWI-confirmed lacunar infarcts. The proportion of mismatch tissue was similar in patients with lacunar infarcts or nonlacunar strokes (32\% versus $36 \%, P=.734)$.

CONCLUSIONS: Whole-brain CTP is superior to NCCT in identifying small ischemic lesions, including lacunar infarcts, in patients presenting with a lacunar syndrome. Perfusion deficits and mismatch are frequent in lacunar infarcts, but larger studies are warranted to elucidate the clinical significance of these CTP findings.

ABBREVIATIONS: $\mathrm{LI}=$ lacunar infarct; TOAST $=$ Trial of Org 10172 in Acute Stroke Treatment; $T$ TTD $=$ time to drain

S mall-vessel disease is common and causes cognitive, psychiatric, and physical disability. ${ }^{1}$ Lacunar infarcts (LIs) are one of the main manifestations of small-vessel disease, accounting for $10 \%-$ $20 \%$ of all ischemic strokes, and they often present with the characteristic classic lacunar syndromes: pure motor hemiparesis, pure sensory stroke, sensorimotor stroke, ataxic hemiparesis, and dysarthria-clumsy hand syndrome. LIs are usually $<1.5 \mathrm{~cm}$ wide and are often located in the territory of deep perforating arteries such as lenticulostriate, thalamoperforant, or paramedian territories. However,

Received November 18, 2014; accepted after revision December 22.

From the Functional Unit of Cerebrovascular Diseases (S.R., X.U., L.S.R., C.L., A.L.-R. S.A., L.O., A.C.) and Department of Radiology (L.S.R., A.L.-R., L.O.), Hospital Clínic, Barcelona, Spain; Institut d'Investigacions Biomèdiques August Pi i Sunyer (X.U., S.A., Á.C.), Barcelona, Spain; and Department of Medicine (Á.C.), School of Medicine, University of Barcelona, Barcelona. Spain.

Please address correspondence to Xabier Urra, MD, PhD, Institute of Neurosciences, 170 Villarroel, 08036 Barcelona, Spain; e-mail: xurra@clinic.cat; @xabierurra

$\equiv$ Indicates article with supplemental on-line table.

Indicates article with supplemental on-line photo.

http://dx.doi.org/10.3174/ajnr.A4303
LIs can also result from in situ occlusion of single superficial perforators from pial arteries. LIs are thought to be related to arteriopathy of small blood vessels in the brain, either because of lipohyalinosis or microatheroma. ${ }^{2}$ Less frequent causes are stenosis of a large vessel or microembolization.

The sensitivity of neuroimaging techniques in acute LI is variable, ranging from $40 \%$ for NCCT scans, ${ }^{3,4} 80 \%$ for MR imaging, ${ }^{5}$ and up to $94 \%$ for DWI. ${ }^{6}$ Conflicting results have been reported regarding the presence of perfusion deficits in patients with $\mathrm{LI},{ }^{7-11}$ with sensitivities varying from $0 \%$ to $68 \%$ in studies using MR imaging ${ }^{12-14}$ and from $17 \%$ to $47 \%$ with CTP. ${ }^{15,16}$ In fact, LI is considered one of the causes of false-negative CTP findings. ${ }^{10,11}$ Some reports suggest that the presence of a perfusion deficit is associated with worse outcome in patients with LIs. ${ }^{8,13}$ Regarding the presence of mismatch, a study using perfusion MR imaging did not find a mismatch between perfusion and diffusion sequences in strokes involving perforating arteries, but these results could be explained by the low resolution of their imaging methods. ${ }^{12}$

In this study, we first assessed the clinical utility of CTP in the 


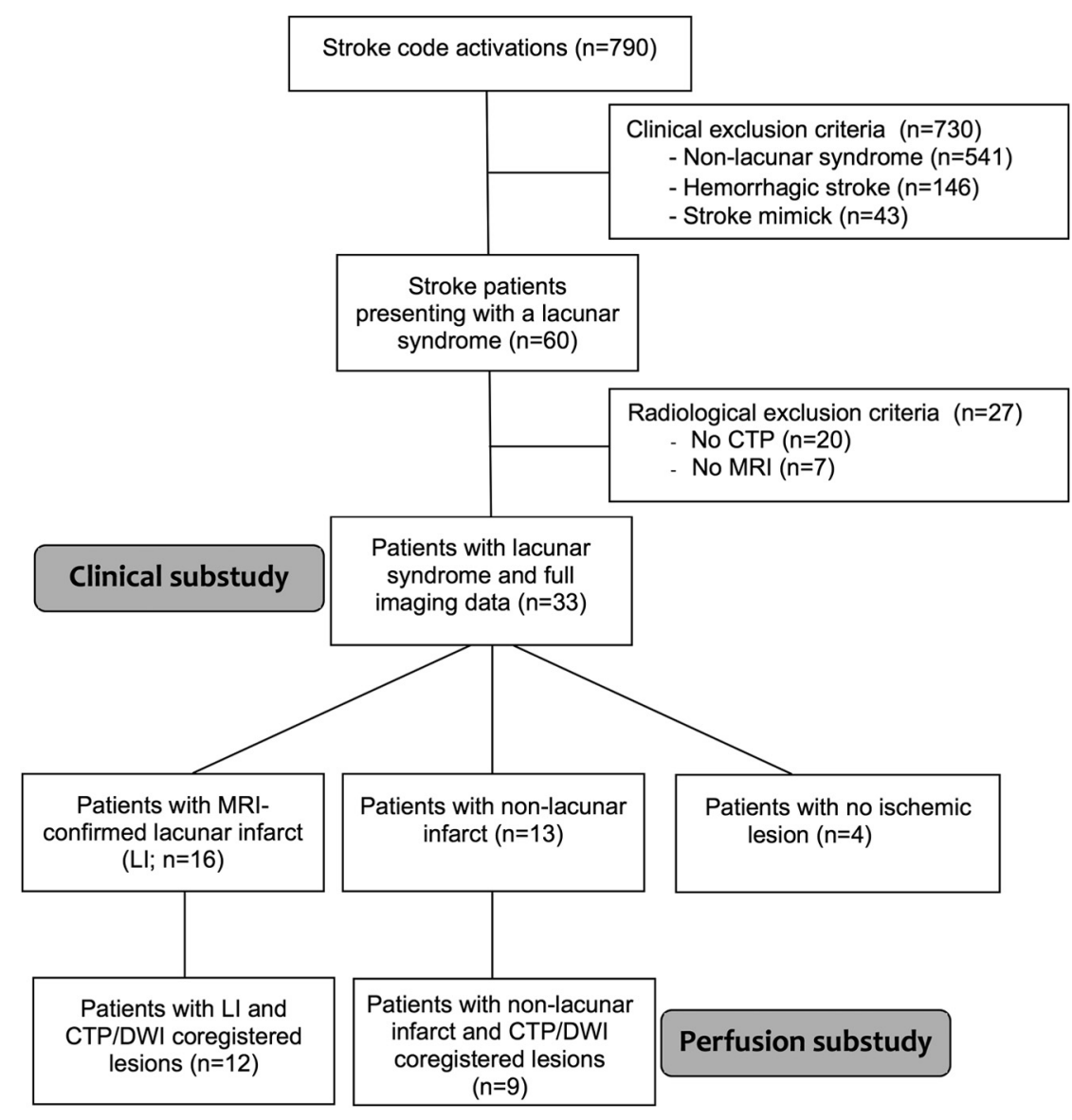

FIG 1. Flow chart describing the patients in the study.

assessed at arrival, at day 1 after admission, at day 7 or discharge, and at day 90 . Functional outcome was assessed by using the mRS at a follow-up visit at 3 months. Because many of the patients were expected to have mild strokes, favorable recovery was defined as an $\mathrm{mRS}$ score of $\leq 1$.

The study protocol was approved by the institutional review board of the center, and the patients or their legal representatives signed a written informed consent if they were treated with $\mathrm{tPA}$ and were older than 80 years of age.

\section{Neuroimaging}

NCCT and CTP were performed at admission at a median (interquartile range) delay of 245 minutes (range, 175344 minutes) after stroke onset on a Somatom Definition Flash 128-section dual-source CT system (Siemens, Erlangen, Germany) with a 98-mm z-coverage and 26 time points acquired at each 1.5 seconds (total acquisition time, 39 seconds). Fifty milliliters of nonionic iodinated contrast was administered intravenously at $5 \mathrm{~mL} / \mathrm{s}$ by using a power injector. CT perfusion imaging parameters were $80 \mathrm{kV}$ (peak), $250 \mathrm{mAs}$, 1.5-second rotation, and $2-\mathrm{mm}$ thickness, and color maps were reformatted at 4-mm

real-life scenario of patients presenting to the emergency department with a lacunar syndrome. Then, we studied the details of the perfusion abnormalities in a subgroup of patients with MR imaging-confirmed LIs and compared them with those of patients with nonlacunar infarcts.

\section{MATERIALS AND METHODS \\ Patients}

Because CTP is routinely used in our institution in the work-up of patients arriving in the first hours after stroke, we studied all patients in the Stroke Unit registry of the Hospital Clínic, Barcelona, admitted from January 2009 to December 2012, in whom the Stroke Code was activated (patients within 8 hours of stroke onset and wake-up strokes). The description of the study population is summarized in Fig 1.

Twenty-four patients (73\%) received treatment with tPA within 4.5 hours after a head NCCT ruled out intracranial hemorrhage, and CTP was performed just before $(n=4)$ or during tPA perfusion $(n=20)$. Stroke etiology was assigned by using the Trial of Org 10172 in Acute Stroke Treatment (TOAST) criteria ${ }^{17}$ after a complete diagnostic work-up. All patients were admitted to an intermediate care Stroke Unit and were treated by stroke neurologists certified in the use of the NIHSS. The demographics, risk factors, clinical course, imaging data, concomitant therapies, and functional outcome were prospectively collected and stored in the Hospital Clínic Stroke Unit data base. The neurologic course was thickness. CTP maps were calculated with syngo CT Neuro Perfusion VA20 (Siemens), which uses singular-value decomposition without delay correction and automatically performs motion correction and selects an arterial input function from an unaffected artery and venous output function from a large draining vein. The perfusion maps generated were the following: CBF, CBV, MTT, time to maximum of residue function maps, TTP, time to drain (TTD), and MIP. Ischemic lesions usually show decreased CBV and $\mathrm{CBF}$ and prolonged measures of time maps (Fig 2A).

Two experienced physicians (a neurologist and a neuroradiologist) evaluated the NCCT and the CTP maps and were only aware of the side of the clinical symptoms but were blinded to the follow-up MR imaging data and clinical outcome. They described the presence of perfusion deficits (with lacunar or nonlacunar appearance) and their location. A consensus reading was achieved in cases of differences in the individual readings.

MR imaging was performed on a $1.5 \mathrm{~T}$ scanner at a median delay of 26 hours (interquartile range, 18-43 hours) after stroke onset. The stroke MR imaging protocol included a DWI sequence, obtained with b-values of 1000, 5-mm section thickness, and $128 \times 128$ matrix. DWI hyperintensities were analyzed in isotropic images. A vascular neurologist (X.U.) confirmed the final diagnosis by reviewing the patient's clinical course and DWI findings and by using Amira software (www.amira.com) segmented the infarct in DWI by a semiautomatic procedure, selecting DWI 


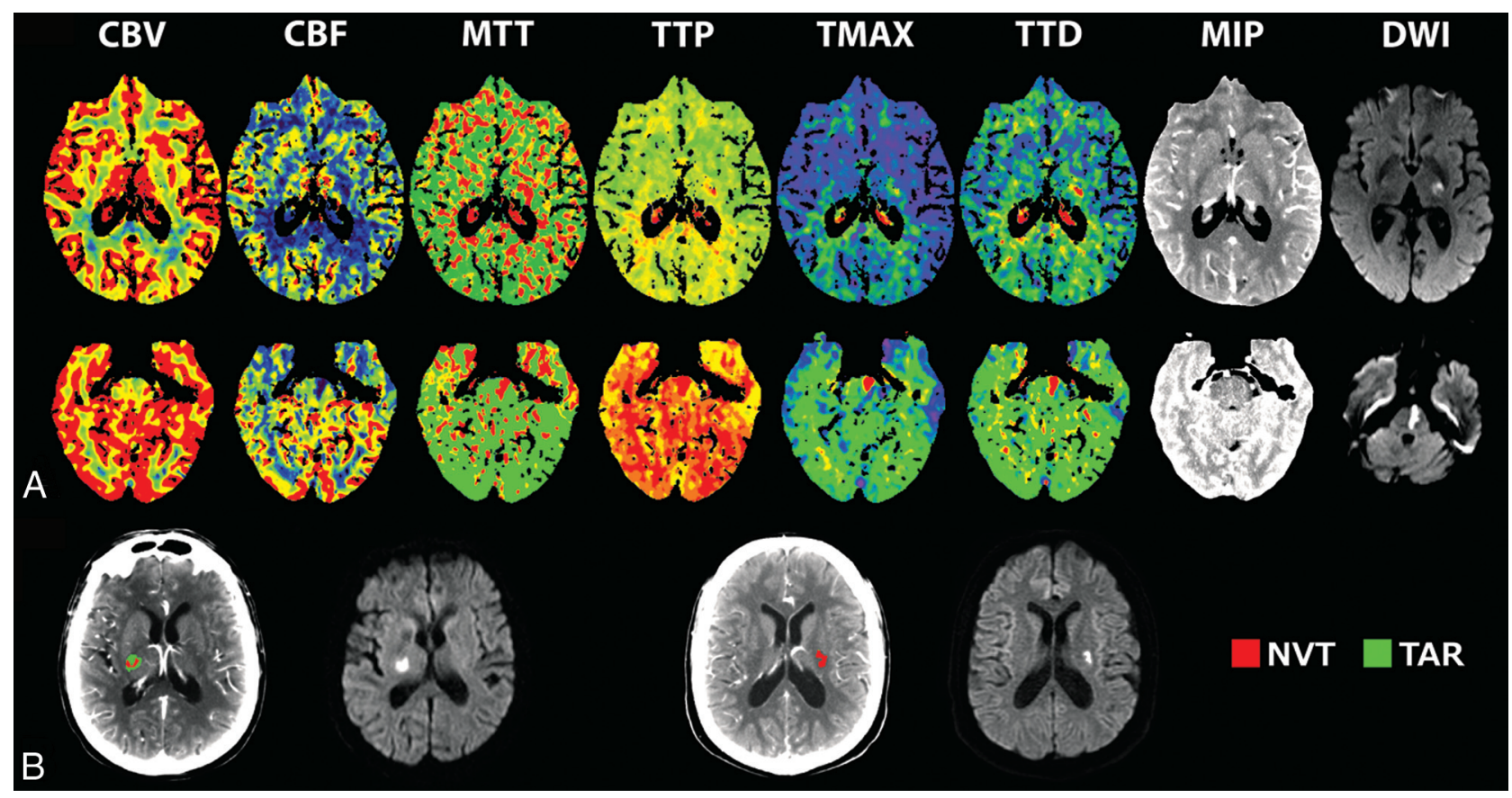

FIG 2. Neuroimaging methods. A, CTP maps and DWI lesions in a patient with a supratentorial lacunar infarct (upper row) and in a patient with a brain stem paramedian infarct (lower row). B, Representative examples of the quantification of NVT and TAR and the final DWI lesion in a patient with mismatch (left) and a patient with no mismatch (right). NVT indicates nonviable tissue; TAR, tissue at risk.

ROIs with a signal intensity exceeding by $>3$ SDs the intensity of the contralateral hemisphere. The volume of the DWI lesion was calculated, and the lesion shape was categorized as previously described. ${ }^{18}$ To be categorized as LI on DWI, the infarcts had to be located within the territories of the lenticulostriate, thalamoperforant, paramedian, or white matter long medullary arteries, and they had to be $<1.767 \mathrm{~cm}^{3}$ (the volume of a sphere with a diameter of $1.5 \mathrm{~cm}) .{ }^{19}$ This strict definition was chosen to describe the perfusion characteristics of a homogeneous group of patients with typical LIs.

For the analysis of the perfusion abnormalities, an image-processing pipeline was developed by using in-house fully automated software running in Matlab (Version 2013a; MathWorks, Natick, Massachusetts) to implement a comprehensive analysis of the perfusion maps. A neurologist (X.U.) evaluated time maps (MTT, time to maximum of residue function maps) and semiautomatically delineated the regions defining perfusion abnormalities. This ROI was then registered to perfusion maps generated by using MIStar (Apollo Medical Imaging Technology, Melbourne, Australia), a software that uses singular-value decomposition with a delay correction. ${ }^{20}$ Within this ROI, we used a range of relative and absolute thresholds in the $\mathrm{CBF}$ and time maps to define infarct core or nonviable tissue and critically hypoperfused or tissue at risk, respectively (Fig 2B). "Mismatch" was defined as a greater extent of tissue at risk than nonviable tissue. ${ }^{21}$ In an effort not to underestimate the size of the lesion defined in time maps, we applied a dilation filter to the ROI. Nonviable tissue or infarct core was segmented on the basis of a relative CBF threshold of $30 \%$ of the value in the contralateral hemisphere, and tissue at risk, on the basis of a delay time of 2 seconds. ${ }^{22}$ Additionally, we explored the presence of mismatch by using various combina- tions of CBF (relative CBF of $<20 \%-40 \%$ ) and delay time thresholds ( $>2$ or 3 seconds).

For the coregistration of the infarct, each perfusion CT map was coregistered to the corresponding 24- to 48-hour DWI. Using Statistical Parametric Mapping (SPM8; http://www.fil.ion. ucl.ac.uk/spm/software/spm8), we automatically subjected the DWI to a sequence of two $3 \mathrm{D}$ registration procedures to match the CTP map by using TOF as an intermediary. The first step was a 6- $d f$ coregistration of the DWI to the TOF. Following this step, we performed another rigid coregistration of the TOF to the CTP, and we finally applied the obtained transformation matrix to the DWI, placing CTP maps, DWI, and TOF in the register. Once CTP and DWI were in the register, we checked that the lesions defined in DWI and CTP were at the same anatomic and spatial locations.

\section{Statistics}

Normal distribution of all studied variables was assessed. Continuous variables were compared with Student $t$, Mann-Whitney, or Kruskal-Wallis tests as appropriate. Correlations were assessed with Spearman coefficients, and categoric variables were compared with the Fisher exact test. Interrater agreement was assessed with the $\kappa$ statistic. The level of significance was established at a 2 -tailed value of $P<.05$. All tests were performed by using SPSS, Version 20.0 (IBM, Armonk, New York).

\section{RESULTS}

\section{Clinical and MR Imaging Characteristics of Patients Presenting with Lacunar Syndrome}

The main clinical characteristics of the study patients are summarized in the Table. Most patients presented with a sensory motor syndrome $(34 \%)$ or a pure motor syndrome (30\%). According to 


\begin{tabular}{lcc} 
Clinical characteristics of the study population & \\
\hline \multicolumn{1}{c}{ Patients } & $\begin{array}{c}\text { Lacunar Syndrome } \\
(\boldsymbol{n}=33)\end{array}$ & $\begin{array}{c}\text { MRI-Confirmed } \\
\text { LI }(\boldsymbol{n}=16)\end{array}$ \\
\hline Age (yr) (mean) (SD) & $65.5(11)$ & $62.6(10)$ \\
Sex (\%) (male/female) & $70: 30$ & $62.5: 37.5$ \\
tPA (\%) & 73 & 62.5 \\
NIHSS score (median) (IQR) & & \\
Admission & $4(3-5.5)$ & $3.5(3-4.5)$ \\
24 Hours & $3(1-5)$ & $3(2-4.5)$ \\
Discharge & $2(1-4)$ & $2.5(1.5-4)$ \\
Day 90 & $1(0-2)$ & $1(0-2)$ \\
Excellent outcome (\%) & 48 & 44 \\
TOAST (\%) & & \\
Lacunar & 64 & 81 \\
Large-artery atherosclerosis & 12 & 0 \\
Cardioembolic & 3 & 0 \\
Undetermined & 21 & 19 \\
\hline
\end{tabular}

Note:-IQR indicates interquartile range.

the TOAST criteria, most of the strokes were related to smallartery occlusions; only 3 patients had brain MR imaging findings consistent with LI but diagnosed as infarcts of undetermined origin due to the coexistence of ipsilateral carotid stenosis of $>50 \%(n=2)$ or atrial fibrillation $(n=1)$. Only 5 patients (2 with MR imaging-confirmed LIs) experienced neurologic deterioration.

On MR imaging, 29 of 33 patients (88\%) had an ischemic lesion on DWI and 4 patients had negative findings on MRI, despite the presence of focal symptoms or signs for $>24$ hours ( 1 pure sensory, 1 pure motor, 1 ataxic hemiparesis, and 1 sensorimotor syndrome). Thirteen patients had non-LIs, and 16 had LIs (additional information can be found in On-line Fig 1 and Online Table 1). These involved the thalamoperforator arteries in 7 patients, the territory of paramedian arteries in 6 , the lenticulostriate territory in 2 , and the white matter long medullary arteries in 1 patient. The median volume of LIs was $0.62 \mathrm{~cm}^{3}$, and most had a tubular $(56 \%)$ or nodular $(37.5 \%)$ shape.

\section{Diagnostic Yield of CTP in Patients with Lacunar Syndrome and MR Imaging-Confirmed LIs}

CTP results matched those of the follow-up MR imaging in 17/33 cases, while NCCT did so in only 6/33 cases. Both the sensitivity and positive predictive value for the symptomatic acute lesions were higher for CTP compared with NCCT (50\% versus 18\%, $P=$ $.023 \%$, and $54 \%$ versus $20 \%, P=.05$, respectively). The negative predictive value was similar for CTP and CT (43\% versus $37.5 \%$, $P=1.0)$, and specificity was the same (20\%). Interrater agreement was also equal for NCCT and CTP $(\kappa=0.61)$. CTP was more sensitive for supratentorial lesions compared with infratentorial lesions (65\% versus $16 \%, P=.011)$. Among the 12 falsepositive cases, in 6 cases, the CTP actually showed small perfusion deficits, but they were greater than the $1.767-\mathrm{cm}^{3}$ limit defined for LIs. In 5 cases, the perfusion maps showed a small hypoperfusion, but the stroke was found to be in another territory on the DWI; and in 1 case, a CTP hypoperfusion had negative findings on follow-up DWI. In the subgroup of 16 cases with MR imagingconfirmed LI, CTP also had superior sensitivity and positive predictive value to NCCT (62.5\% versus $19 \%, P=.029 \%$, and $83 \%$ versus $43 \%, P=.129$, respectively). The proportion of CTP studies with negative findings was not significantly different between patients with lacunar syndrome treated and not treated with tPA

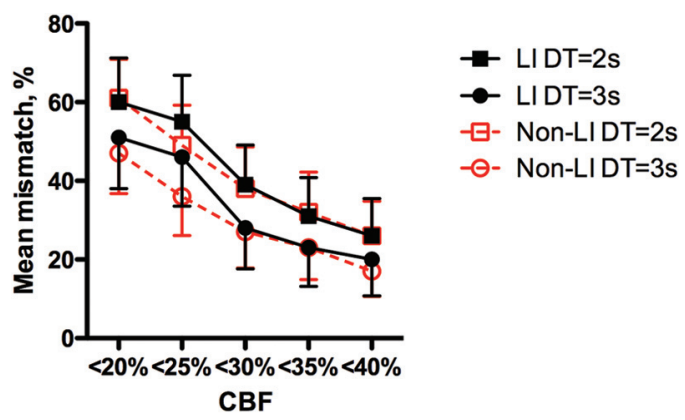

FIG 3. Rates of mismatch tissue according to different definitions of nonviable tissue and tissue at risk in patients with $\mathrm{LI}$ and nonlacunar infarcts. Values are mean and standard error of the mean. DT indicates delay time.

(39.4\% and $12.1 \%, P=.59)$ or in the LI group $(37.5 \%$ and $18.8 \%$, $P=.55)$.

Although the evaluators assessed all perfusion maps together, the infarcts were best seen on TTD maps, which often showed the LIs as small red dots that were easy to distinguish from the background (On-line Fig 2). Among the 16 cases with MR imagingconfirmed LI, CTP with negative findings was due to movement artifacts in 2 patients and to lesions located in the brain stem in 4 patients. In 2 of them, small perfusion deficits could be seen after reviewing the DWI, but the lesions were either too small or poorly differentiated from the background.

\section{Perfusion Deficits in Patients with MR Imaging-Confirmed LIs and in Other Strokes}

In the nonblinded analysis, a clear perfusion abnormality to be segmented was identified in 12/16 of LIs and in 8/12 of nonlacunar infarcts within the ROIs of lesions on DWI. Mismatch was found in 5 (42\%) of the patients with measurable perfusion deficits by using the standard thresholds, and the mean percentage of mismatch volume ranged from $20 \%$ by using the strictest thresholds to $65 \%$ in both patients with MR imaging-confirmed LI and those with nonlacunar strokes (Fig 3). The proportion of mismatch patients ranged from $17 \%$ to $50 \%$ in patients with LIs and from $11 \%$ to $56 \%$ in patients with nonlacunar strokes.

Although clinical progression was more frequent in patients without mismatch (43\% versus $0 \%, P=.205)$, the rate of improvement after thrombolysis and functional outcome at 3 months and the rest of the clinical features were similar between patients with and without mismatch (data not shown).

\section{DISCUSSION}

Previous studies have reported conflicting results regarding perfusion abnormalities in patients with lacunar infarcts, probably reflecting differences in available technology. The results of this study by using whole-brain-coverage CTP are very similar to those of a recent study by using perfusion MR imaging in patients with LI that reported perfusion deficits in $10 / 16$ patients. ${ }^{14}$ The sensitivity of whole-brain CTP was much higher than that of NCCT for acute stroke and in particular for LI, and the greater sensitivity of CTP in this study may reflect both whole-brain coverage of perfusion maps and the refinement of current parameters to assess perfusion maps. Studies with negative findings were due to very small lesions that were poorly differentiated from the 
background, especially in the brain stem; and in a few cases, they were due to poor image quality from movement artifacts. In studies with no movement artifacts and after reviewing the results of the DWI, we could identify a clear perfusion deficit in most patients with LIs.

The rate of clinical deterioration was rather low in patients with LIs, and all patients with clinical deterioration had a nomismatch profile. However, the number of patients with MR imaging-confirmed LIs was too low to draw definite conclusions on the clinical consequences of the perfusion deficits in these patients. Larger studies will be required to establish whether the presence of mismatch in LIs represents a predictor of clinical recovery as it did for nonlacunar strokes, ${ }^{23}$ particularly after the administration of thrombolytic therapy. ${ }^{24}$ The specificity of CTP to show MR imaging-confirmed lacunar stroke was low because some small hypoperfused lesions were not confirmed to be true LIs by using a strict DWI volume limit, but sensitivity was higher and a whole-brain-coverage CTP in the first hours after symptom onset may be helpful in evaluating patients in whom the diagnosis is not clear, even when the symptoms are mild.

TTD maps provided high-contrast images that were most useful in identifying small perfusion deficits. TTD is a recently introduced time-related perfusion parameter defined as the sum of the time from arterial enhancement to tissue enhancement and MTT. ${ }^{25}$ Condensing both pathologic changes in contrast bolus delay time from arterial enhancement to tissue enhancement and tissue transit time, TTD describes the time of contrast medium washout and is very sensitive to all kinds of hemodynamic disturbances. $^{26}$

It has been argued that patients with true LIs should not have detectable perfusion deficits and/or that they should have no mismatch between infarcted tissue and hypoperfused tissue. ${ }^{12}$ This study suggests that the hemodynamic abnormalities of LIs, though small, are very similar to those described in infarcts resulting from occlusion of greater vessels (it has been claimed that lacunes are just small strokes). ${ }^{27}$ The hypoperfusion in LIs may reflect the fact that occlusions of small arterioles are not compensated by dilation of neighboring arterioles. ${ }^{28}$ Although there are contradictory statements in the literature regarding intraparenchymal arteriolar-to-arteriolar anastomoses, ${ }^{29}$ the capillary bed of the brain comprises an attenuated network of intercommunicating vessels, and dilation in capillaries after penetrating arteriole occlusion in the rat brain allows some collateral flow from one arteriolar territory to neighbor territories. ${ }^{28}$ This could result in less severely hypoperfused areas with mismatch in CTP maps.

In addition to hypoperfusion, other mechanisms may contribute to the final infarct after occlusion of small vessels. In experimental models, these lesions are accompanied by activation of inflammatory cells, ${ }^{30}$ and extensive increases in vascular permeability have been described in patients with small-vessel disease and in particular LI. ${ }^{31}$ Although we have seen patients in whom the final infarct was greater than the initial hypoperfused area, we were not able to accurately measure the initial ischemic volume, and therefore lesion expansion, because the segmentation of the perfusion abnormalities was focused on delineating the hypoperfusion within the DWI lesion.

The main limitation of this study is its small sample size, de- spite studying all consecutive patients arriving at our center for the 3 years in which the Stroke Code was activated. The low percentage of patients with a lacunar syndrome among them is probably due to the lower clinical severity of these strokes. CTP was not performed in 20 patients because acute revascularization therapies were not considered in patients with milder symptoms. Therefore, these findings cannot be extrapolated to patients with other clinical presentations or to LIs assessed more protractedly after the onset of symptoms, given the dynamic nature of perfusion data after acute stroke. The small size of LIs is also challenging for CTP-DWI coregistration, but its accuracy was manually confirmed. Thus, we do not think that the results are less accurate than those in nonlacunar infarcts.

\section{CONCLUSIONS}

These results suggest that whole-brain CTP technology can identify abnormal perfusion maps in $62.5 \%$ of patients with DWIconfirmed LI, mostly in supratentorial lesions. The rate of mismatch in patients with perfusion deficits was similar to that in patients with other strokes, suggesting that some collateral flow exists in human microcirculation after the occlusion of small penetrating vessels. However, further study is warranted to elucidate whether the presence of mismatch in patients with LI predicts a better clinical outcome and might even be used to select candidates for new therapies, for example, to receive thrombolytic therapy after the established 4.5-hour window.

\section{ACKNOWLEDGMENTS}

We thank Dr Martha Vargas for her assistance in developing the patient data base.

\section{REFERENCES}

1. Wardlaw JM, Smith C, Dichgans M. Mechanisms of sporadic cerebral small vessel disease: insights from neuroimaging. Lancet $\mathrm{Neu}$ rol 2013;12:483-97

2. Arboix A, Martí-Vilalta JL. Lacunar stroke. Expert Rev Neurother 2009;9:179-96

3. Chamorro A, Sacco RL, Mohr JP, et al. Clinical-computed tomographic correlations of lacunar infarction in the Stroke Data Bank. Stroke 1991;22:175-81

4. Arboix A, Martí-Vilalta JL, García JH. Clinical study of 227 patients with lacunar infarcts. Stroke 1990;21:842-47

5. Hommel M, Besson G, Le Bas JF, et al. Prospective study of lacunar infarction using magnetic resonance imaging. Stroke 1990;21:546-54

6. Schonewille WJ, Tuhrim S, Singer MB, et al. Diffusion-weighted MRI in acute lacunar syndromes: a clinical-radiological correlation study. Stroke 1999;30:2066-69

7. Reichenbach JR, Röther J, Jonetz-Mentzel L, et al. Acute stroke evaluated by time-to-peak mapping during initial and early follow-up perfusion CT studies. AJNR Am J Neuroradiol 1999;20:1842-50

8. Yamada M, Yoshimura S, Kaku Y, et al. Prediction of neurologic deterioration in patients with lacunar infarction in the territory of the lenticulostriate artery using perfusion CT. AJNR Am J Neuroradiol 2004;25:402-08

9. Kane I, Hand PJ, Rivers C, et al. A practical assessment of magnetic resonance diffusion-perfusion mismatch in acute stroke: observer variation and outcome. J Neurol 2009;256:1832-38

10. Campbell BC, Weir L, Desmond PM, et al. CT perfusion improves diagnostic accuracy and confidence in acute ischaemic stroke. J Neurol Neurosurg Psychiatry 2013;84:613-18

11. Biesbroek JM, Niesten JM, Dankbaar JW, et al. Diagnostic accuracy 
of CT perfusion imaging for detecting acute ischemic stroke: a systematic review and meta-analysis. Cerebrovasc Dis 2013;35:493-501

12. Gerraty RP, Parsons MW, Barber PA, et al. Examining the lacunar hypothesis with diffusion and perfusion magnetic resonance imaging. Stroke 2002;33:2019-24

13. Poppe AY, Coutts SB, Kosior J, et al. Normal magnetic resonance perfusion-weighted imaging in lacunar infarcts predicts a low risk of early deterioration. Cerebrovasc Dis 2009;28:151-56

14. Förster A, Kerl HU, Wenz H, et al. Diffusion- and perfusionweighted imaging in acute lacunar infarction: is there a mismatch? PLoS One 2013;8:e77428

15. Mayer TE, Hamann GF, Baranczyk J, et al. Dynamic CT perfusion imaging of acute stroke. AJNR Am J Neuroradiol 2000;21:1441-49

16. Maruya J, Yamamoto K, Ozawa T, et al. Simultaneous multi-section perfusion CT and CT angiography for the assessment of acute ischemic stroke. Acta Neurochir (Wien) 2005;147:383-91; discussion 391-92

17. Adams HP Jr, Bendixen BH, Kappelle LJ, et al; TOAST Investigators. Classification of subtype of acute ischemic stroke: definitions for use in a multicenter clinical trial. Stroke 1993;24:35-41

18. Hervé D, Mangin JF, Molko N, et al. Shape and volume of lacunar infarcts: a 3D MRI study in cerebral autosomal dominant arteriopathy with subcortical infarcts and leukoencephalopathy. Stroke 2005;36:2384-88

19. Gerraty RP, Parsons MW, Alan Barber P, et al. The volume of lacunes. Stroke 2001;32:1937-38

20. Bivard A, Levi C, Spratt N, et al. Perfusion CT in acute stroke: a comprehensive analysis of infarct and penumbra. Radiology 2013;267:543-50

21. Obach V, Oleaga L, Urra X, et al. Multimodal CT-assisted thrombolysis in patients with acute stroke: a cohort study. Stroke 2011; 42:1129-31
22. Campbell BC, Christensen S, Levi CR, et al. Cerebral blood flow is the optimal CT perfusion parameter for assessing infarct core. Stroke 2011;42:3435-40

23. Wechsler LR. Imaging evaluation of acute ischemic stroke. Stroke 2011;42:S12-15

24. Kidwell CS, Alger JR, Saver JL. Evolving paradigms in neuroimaging of the ischemic penumbra. Stroke 2004;35(11 suppl 1):2662-65

25. Thierfelder KM, Sommer WH, Baumann AB, et al. Whole-brain CT perfusion: reliability and reproducibility of volumetric perfusion deficit assessment in patients with acute ischemic stroke. Neuroradiology 2013;55:827-35

26. Abels B, Klotz E, Tomandl BF, et al. Perfusion CT in acute ischemic stroke: a qualitative and quantitative comparison of deconvolution and maximum slope approach. AJNR Am J Neuroradiol 2010;31:1690-98

27. Millikan C, Futrell N. The fallacy of the lacune hypothesis. Stroke 1990;21:1251-57

28. Nishimura N, Rosidi NL, Iadecola C, et al. Limitations of collateral flow after occlusion of a single cortical penetrating arteriole. J Cereb Blood Flow Metab 2010;30:1914-27

29. Moody DM, Bell MA, Challa VR. Features of the cerebral vascular pattern that predict vulnerability to perfusion or oxygenation deficiency: an anatomic study. AJNR Am J Neuroradiol 1990;11:431-39

30. Nishimura N, Schaffer CB. Big effects from tiny vessels: imaging the impact of microvascular clots and hemorrhages on the brain. Stroke 2013;44:S90-92

31. Wardlaw JM, Doubal FN, Valdes-Hernandez M, et al. Blood-brain barrier permeability and long-term clinical and imaging outcomes in cerebral small vessel disease. Stroke 2013;44:525-27 